\title{
Influence of glycosylation on the structure of human natural killer cell receptor NKp30 in complex with its tumor ligand B7-H6
}

\author{
T. Skálová ${ }^{1}$, O. Skořepa ${ }^{2}$, B. Kalousková2 ${ }^{2}$ J. Bláha ${ }^{2}$, C. Abreu², J. Dohnálek ${ }^{1}$, O. Vaněk ${ }^{2}$ \\ ${ }^{1}$ Institute of Biotechnology of the Czech Academy of Sciences, v.v.i., Prümyslová 595, 252 50, Vestec, Czech Republic, \\ ${ }^{2}$ Department of Biochemistry, Faculty of Science, Charles University Prague, Hlavova 8, 12840 Praha, Czech Republic \\ t.skalova@gmail.com
}

NKp30 is an activating receptor on the surface of human natural killer (NK) cells. Its crystal structure has been published previously by Joyce et al. [1], PDB code 3NOI. B7-H6 is an activating immunoligand expressed by some tumor cells. Its structure in complex with NKp30 has been described by Li et al. [2], PDB code 3PV6.

In this study, we present a new crystal structure of NKp30:B7-H6 at resolution $3.1 \AA$ using homogenously glycosylated proteins produced in HEK293S GnTI' cell lines. The structure has been deposited in the Protein Data Bank under code 6YJP and published [3].

For the structural study, NKp30 was used with complete glycosylation, while B7-H6 was deglycosylated after the first GlcNAc for better crystallization. The new structure showed the same NKp30:B7-H6 interaction interface as observed by Li et al. (3PV6). Similarly, as in the structure of Joyce et al. (3NOI), NKp30 form dimers. However, the dimers of glycosylated NKp30 are different (the glycan presence hinders the formation of the dimers observed in PDB 3NOI), and according to Pisa server validation, the new dimers are more likely biologically relevant.

Furthermore, the asymmetric contains a dimer of NKp30 (contacts of chains A-C and Bpaper [3] shows a hypothesis B7-H6 ligands during contact

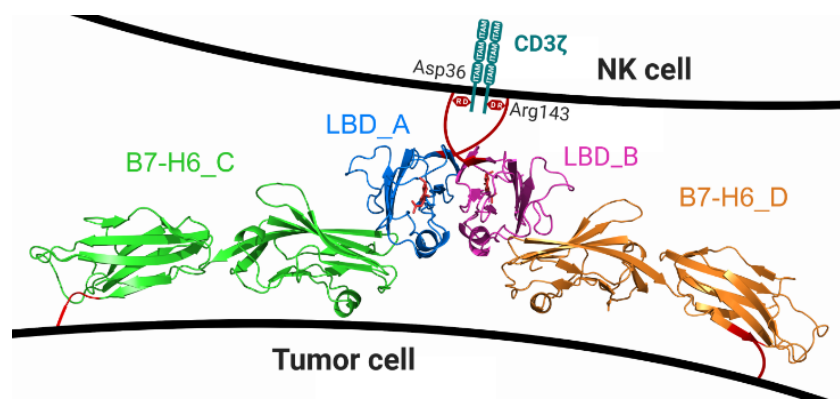

unit of the new crystal structure placed among two B7-H6 molecules $\left.\mathrm{D}_{\text {symm }}\right)$. The illustration taken from our of NKp30 dimer bound between two of the NK cell and the tumor cell.

[1] Joyce, M.G., Tran, P., Zhuravleva, M.A., Jaw, J., Colonna, M., Sun, P.D. (2011) Proc. Natl. Acad. Sci. USA 108, $6223-6228$.

[2] Li, Y., Wang, Q., Mariuzza, R.A. (2011). J. Exp. Med. 208, 703-714.

[3] Skořepa, O., Pazicky, S., Kalousková, B., Bláha, J., Abreu, C., Ječmen, T., Rosůlek, M., Fish, A., Sedivy, A., Harlos, K., Dohnálek, J., Skálová, T., Vaněk, O. (2020). Cancers 12, 1998.

\section{Keywords: NK cell; NKp30; B7-H6; glycosylation; oligomerization}

This research was funded by Czech Science Foundation (18-10687S), MEYS of the Czech Republic (LTC17065, CZ.02.1.01/0.0/0.0/16 013/0001776), BIOCEV (ERDF CZ.1.05/1.1.00/02.0109), and Charles University (GAUK 927916, SVV 260427/2020). CIISB research infrastructure project LM2015043, funded by MEYS CR, is gratefully acknowledged for the financial support of experiments at the CMS. The authors also acknowledge the support and the use of Instruct-ERIC resources (PID: 1314) and iNEXT (PID: 2322) infrastructures. The Wellcome Centre for Human Genetics is supported by Wellcome Trust grant 203141/Z/16/Z. O.S. and O.V. received short-term scientific mission support from COST Action CA15126. 